\title{
Impulsive State Feedback Control of Cheese Whey Fermentation for Single-Cell Protein Production
}

\author{
Chunjin Wei, ${ }^{1}$ Shuwen Zhang, ${ }^{1}$ and Lansun Chen ${ }^{2}$ \\ ${ }^{1}$ Science College, Jimei University, Xiamen, Fujian 361021, China \\ ${ }^{2}$ Academy of Mathematics and System Sciences, Chinese Academy of Sciences, Beijing 100080, China \\ Correspondence should be addressed to Chunjin Wei; chunjinwei92@163.com
}

Received 22 March 2013; Accepted 18 June 2013

Academic Editor: Yongkun Li

Copyright (c) 2013 Chunjin Wei et al. This is an open access article distributed under the Creative Commons Attribution License, which permits unrestricted use, distribution, and reproduction in any medium, provided the original work is properly cited.

\begin{abstract}
The work is the analysis of a mathematical model of cheese whey fermentation for single-cell protein production with impulsive state feedback control. Through the analysis, the sufficient conditions of existence and stability of positive order-1 periodic solution are obtained. It is shown that the system either tends to a stable state or has a periodic solution, which depends on the feedback state, the control parameter of the dilution rate, and the initial concentrate of microorganism and substrate. For some special cases, it is also shown that the system may exist in order-2 periodic solution. Furthermore, our findings are confirmed by means of numerical simulations.
\end{abstract}

\section{Introduction}

Single-cell protein (SCP) is the protein extracted from cultivated microbial biomass. It can be used for protein supplementation of a staple diet by replacing costly conventional sources like soymeal and fishmeal to alleviate the problem of protein scarcity. Increasing demand for protein sources of high nutrition value have stimulated the application of singlecell proteins, yeast, bacteria, and algae in human foods or animal feeds [1]. The interest in agricultural and industrial wastes as substrates for the production of single-cell proteins has increased recently (see [2-5] and the references therein). For example, Kurbanoglu and Algur [6] studied single-cell protein production from ram horm hydrolysate by bacteria, and El-Saadany et al. [7] studied the production of single cell protein from agricultural wastes by fungi.

The production of microbial biomass is done mainly by a continuous fermentation system [8]. According to different reactions and differential control technologies, many dynamic models concerning the culture of microorganisms in the continuous bioreactor have been established [9-13]. However, there are a lot of factors affecting the growth and reproduction of the microorganisms in the process of bioreacts [14]. For example, for some aerobic microorganisms, the dissolved oxygen (DO) is a key factor to the growth of the aerobic microorganisms. In order to maintain the dissolved oxygen concentration in an appropriate range, it is necessary to regulate the microorganisms' concentration to not exceed a set level [15].

As shown in Figure 1, if the microorganisms' concentration reaches the preset value, the valve is opened by the photoelectric control devices, and a certain amount of regulator solution is input. Then, the microorganisms' concentration is decreased and less than the preset value. At this time, the valve is closed by the control system until the next moment at which the microorganisms' concentration reaches the preset value once again. Therefore, the microorganism concentration can be decreased through the control methods such as replenishing water or other regulator solution to reduce the concentration of the microorganism.

In recent years, in order to obtain the optimal conditions for microorganism growth and decrease the inhibition of the microorganism concentration or other negative effects, many authors introduced the state-dependent impulsion into the microorganism culture process and analyzed the system's dynamic behavior [16-18]. However, so far, no papers have discussed the mathematical model of cheese whey fermentation for single-cell protein production with impulsive feedback control. 


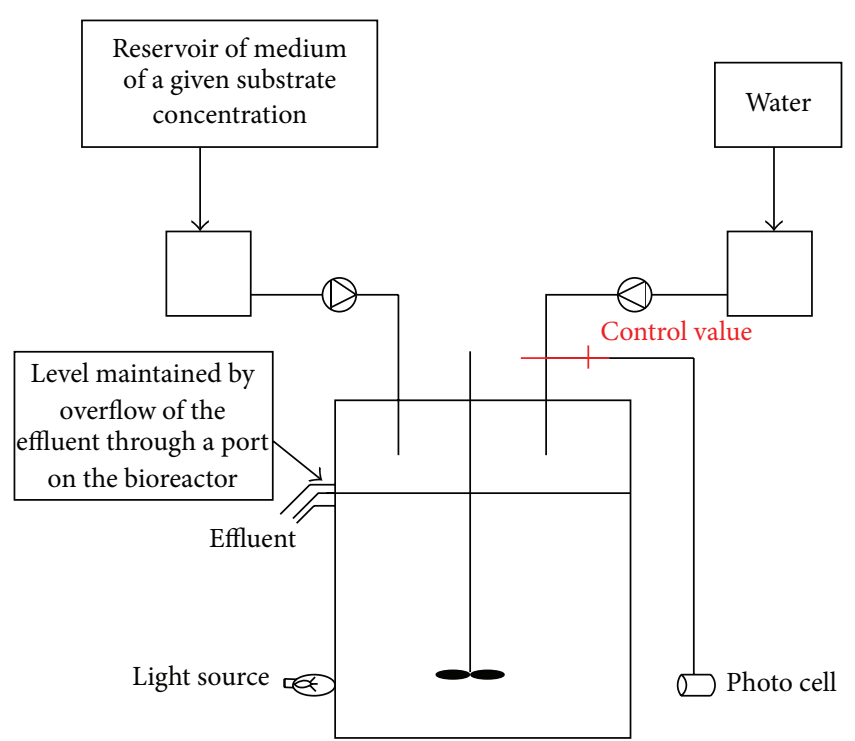

FIGURE 1: Schematic diagram of microorganism culture system with feedback control.

This paper aims to propose a mathematical model of cheese whey fermentation for single-cell protein production with feedback control. We will discuss the existence and stability of periodic solution of the system with impulsive feedback control by using the existence criteria [19] and the stability theorem [20]. The paper is organized as follows. The mathematical model and some preliminary results are introduced in Section 2. In Section 3, the qualitative analysis of the system without impulsive effect is given. In Section 4, the existence and stability of order-1 periodic solution of the system with impulsive state feedback control are investigated. Numerical simulations and some discussion are provided in Section 5 .

\section{Model Formulation}

Ghaly et al. [8] proposed the following mathematical model of cheese whey fermentation for the production of single-cell protein:

$$
\begin{gathered}
S^{\prime}(t)=-\frac{\mu S x}{\delta_{1}\left(K_{s}+S\right)}-m x+D\left(S_{i}-S\right), \\
x^{\prime}(t)=\frac{\mu S x}{K_{s}+S}-\left(K_{d}+D\right) x,
\end{gathered}
$$

where $x$ denotes the concentration of the microorganism $(\mathrm{g} / \mathrm{L})$ and $S$ denotes the concentration of the limiting substrate $(\mathrm{g} / \mathrm{L})$ in the bioreactor at time $t . S_{i}$ denotes the influent substrate concentration $(\mathrm{g} / \mathrm{L}) . D$ is the dilution rate; $\mu$ is the maximum special growth rate; $K_{s}$ is the saturation constant, equal to the substrate concentration at one-half the maximum special growth rate $(\mathrm{g} / \mathrm{L}) ; K_{d}$ is the endogenous microbial decay coefficient $\left(h^{-1}\right) ; \delta_{1}$ is the yield coefficient; $m$ is the maintenance coefficient; and $m x$ denotes the substrate uptake rate for the microorganism maintenance.
In some cases, if the concentration of microorganism is lower than a critical value (a preset value given by experiments), it is not necessary to take the control measures. But if the concentration reaches the critical value, some negative effects such as airborne inhibition and production inhibition and so on may happen, and the control methods such as liquefying medium and replenishing water should be taken to decrease the concentration of the microorganism. Once the concentration is decreased, it also should take some time to reach the critical value. Therefore, the system (1) can be modified as follows by introducing the impulsive state feedback control:

$$
\begin{gathered}
S^{\prime}(t)=-\frac{\mu S x}{\delta_{1}\left(K_{s}+S\right)}-m x+D\left(S_{i}-S\right) \\
x^{\prime}(t)=\frac{\mu S}{K_{s}+S} x-\left(K_{d}+D\right) x, \quad x<h, \\
\Delta S(t)=-b S, \quad x=h, \\
\Delta x(t)=-b x, \quad x(0)=x_{0}<h,
\end{gathered}
$$

where $\Delta x=x\left(t^{+}\right)-x(t), \Delta S=S\left(t^{+}\right)-S(t), 0<b<1$, is the fraction of the concentration of microorganism and substrate that decreases due to the feedback control when the microorganism concentrate $x$ reaches the critical value $h$.

In the following, we mainly discuss the existence of periodic solution of (2) by the existence criteria of the general impulsive autonomous system. Before starting our main results, we give some topological definitions about impulsive differential equations.

Definition 1 (Laksmikantham et al. [21]). A triple $\left(X, \pi, R_{+}\right)$ is said to be a semidynamical system if $X$ is metric space, $R_{+}$ is the set of all nonnegative reals, and $\pi: X \times R_{+} \rightarrow X$ is a continuous function such that

(i) $\pi(x, 0)=x$ for all $x \in X$;

(ii) $\pi(\pi(x, t), s)=\pi(x, t+s)$ for all $x \in X$ and $t, s \in R_{+}$.

We denote sometimes a semidynamical system $\left(X, \pi, R_{+}\right)$ by $(X, \pi)$.

For any $x \in X$, the function $\pi_{x}: R_{+} \rightarrow X$ defined by $\pi_{x}(t)=\pi(x, t)$ is continuous, and we call $\pi_{x}$ the trajectory of $x$. The set

$$
C^{+}(x)=\left\{\pi(x, t) \mid t \in R_{+}\right\}
$$

is called the positive orbit of $x$. For any subset $M$ of $X$, we let

$$
M^{+}(x)=C^{+}(x) \cap M-x, \quad M^{-}=G(x) \cap M-x,
$$

where

$$
\begin{gathered}
G(x)=\cup\left\{G(x, t) \mid t \in R_{+}\right\}, \\
G(x, t)=\{y \mid \pi(y, t)=x\}
\end{gathered}
$$

is the attainable set of $x$ at $t \in R_{+}$. Finally, we set $M(x)=$ $M^{+}(x) \cup M^{-}(x)$. 
Definition 2 (Laksmikantham et al. [21]). An impulsive semidynamical system $(X, \pi ; M, I)$ consists of a semidynamical system $(X, \pi)$ together with a nonempty closed subset $M$ of $X$ and a continuous function $I: M \rightarrow X$ such that the following properties hold:

(i) no point $x \in X$ is a limit point of $M(x)$,

(ii) $[t \mid G(x, t) \cap M \neq \emptyset]$ is a closed subset of $R_{+}$.

We write $N=I(M)=\{y \in X \mid y=I(x), x \in M\}$ and for any $x \in X, I(x)=x^{+}$. We call $M$ the impulsive set and $I$ the impulsive function.

We define a function $\Phi: X \rightarrow R_{+} \cup\{\infty\}$ as follows:

$$
\Phi(x)= \begin{cases}\infty & \text { if } M^{+}(x)=\phi, \\ s & \text { if } \pi(x, t) \notin M \text { for } 0<t<s, \pi(x, s) \in M\end{cases}
$$

here, we call $s$ the time without impulse of $x$, that is $s$ is the first time when $\pi(x, 0)$ hits $M$.

Definition 3 (Laksmikantham et al. [21]). Let $(X, \pi ; M, I)$ be an impulsive semidynamical system, and let $x \in X$ and $x \notin$ $M$. The trajectory of $x$ is a function $\widetilde{\pi}_{x}$ defined on subset $[0, s)$ of $R_{+}$( $s$ may be $\infty$ ) to $X$ inductively as follows:

$$
\tilde{\pi}_{x}(t)=\pi\left(x_{n+1}^{+}, t\right), \quad \tau_{n-1} \leq t \leq \tau_{n},
$$

where $\left\{x_{n}\right\}$ is the sequence of impulse points of $x$, which satisfied $\pi\left(x_{n-1}^{+}, \Phi\left(x_{n-1}^{+}\right)\right)=x_{n} \cdot \tau_{n}$ is the sequence of time of impulses relative to $\left\{x_{n}\right\}, \tau_{n}=\sum_{k=0}^{n-1} \Phi\left(x_{k}^{+}\right)$.

Definition 4 (Laksmikantham et al. [21]). A trajectory $\tilde{\pi}_{x}$ is said to be periodic of period $\tau$ and order $k$ if there exist positive integers $m \geq 1$ and $k \geq 1$ such that $k$ is the smallest integer for which $x_{m}^{+}=x_{m+k}^{+}$and $\tau=\sum_{i=m}^{m+k-1} \Phi\left(x_{i}^{+}\right)$.

Theorem 5 (Brouwer's fixed-point theorem (Griffel [22])). Every continuous mapping of a closed bounded convex set in $R^{n}$ into itself has a fixed point.

Zeng et al. [19] have proved the existence criteria of periodic solution of an impulsive autonomous system by means of Browers' fixed-point theorem. For convenience of reading, we repeat the main results as follows.

Consider the following general autonomous impulsive differential equations:

$$
\begin{aligned}
& \frac{d x(t)}{d t}=P(x, y) \\
& \frac{d y(t)}{d t}=Q(x, y) \quad(x, y) \notin M, \\
& \Delta x=I_{1}(x, y) \\
& \Delta y=I_{2}(x, y)
\end{aligned} \quad(x, y) \in M .
$$

Here, $(x, y) \in R^{2}, P, Q, I_{1}$, and $I_{2}$ are all functions mapping $R^{2}$ into $R$, and $M \subset R^{2}$ is the set of impulse, and we assume

(H1) $P(x, y), Q(x, y)$ are all continuous with respect to $x$, $y$ in $R^{2}$;

(H2) $M \subset R^{2}$ is a line, and $I_{1}(x, y)$ and $I_{2}(x, y)$ are linear functions of $x$ and $y$.

For each point, $S(x, y) \in M$, and we define that $I$ : $R^{2} \rightarrow R^{2}$ :

$$
\begin{gathered}
I(S)=S^{+}=\left(x^{+}, y^{+}\right) \in R^{2}, \\
x^{+}=x+I_{1}(x, y), \quad y^{+}=y+I_{2}(x, y) .
\end{gathered}
$$

Obviously, $N=I(M)$ is also a line of $R^{2}$ or a subset of a line, and we assume that $N \cap M=\phi$. From Definition 2, we know that (8) is an impulsive semidynamical system. The following theorem gives the conditions on which (8) has an order-1 periodic solution defined by Definition 4 .

Theorem 6 (see [19]). If system (8) satisfies assumptions (H1) and (H2), there exists a bounded closed simply connected region $D$ which has the following properties:

(i) there is no singularity in it, and the boundary $\partial D$ of $D$ satisfies $(D-\partial D) \cap M=\emptyset$;

(ii) $L_{1}=D \cap M$ cannot be tangent with trajectories of (8) except at end points and $I\left(L_{1}\right) \subset D$;

(iii) trajectories with initial point in $\partial D-L_{1}$ will enter into interior of $D$; then, there must exist an order-1 periodic solution of system (8) in region $D$.

\section{Qualitative Analysis of System (1)}

Before discussing the periodic solution of system (2), we should consider the qualitative characteristic of system (2) without impulsive effect, that is, consider the qualitative characteristic of system (1).

Lemma 7. The system (1) is uniformly bounded.

Proof. From the first equation of system (1), we have

$$
S^{\prime}(t)=-\frac{\mu S x}{\left(K_{s}+S\right) \delta_{1}}-m x+D\left(S_{i}-S\right)
$$

The isocline $S^{\prime}(t)=0$, that is, $x=D\left(S_{i}-S\right)\left(K_{s}+S\right) \delta_{1} /((\mu+$ $\left.\left.m \delta_{1}\right) S+m K_{s} \delta_{1}\right)$, which intersects the $S$-axis at points $\left(-K_{s}, 0\right)$ and $\left(S_{i}, 0\right)$, and $x<D\left(S_{i}-S\right)\left(K_{s}+S\right) \delta_{1} /\left(\left(\mu+m \delta_{1}\right) S+m K_{s} \delta_{1}\right)$, $S^{\prime}(t)>0$. Furthermore, $x \rightarrow+\infty$, when $S \rightarrow-\left(m \delta_{1} K_{s} /(\mu+\right.$ $\left.m \delta_{1}\right)$ ) from the right; therefore, there exists $S=\delta$ and $-K_{s}<$ $\delta<-\left(m \delta_{1} K_{s} /\left(\mu+m \delta_{1}\right)\right)$ such that $S^{\prime}(t)>0$. Let the straight line $S=S_{i}$, and $S=\delta$ intersect $x=0$ at the points $A\left(S_{i}, 0\right)$, $D(\delta, 0)$, respectively. Define a function $V(S, x)=S+\left(1 / \delta_{1}\right) x-$ $K$, where $K>S_{i}$. The function $V(S, x)$ intersects the line $S=S_{i}$ and $S=\delta$ at the points $B$ and $C$, respectively. Then, we have $\left.(d V / d t)\right|_{B C}=-\left(D x(t) / \delta_{1}\right)-\left(K_{d} x(t) / \delta_{1}\right)-m x(t)+$ $D\left(S_{i}-S(t)\right)=-\left(K_{d} x(t) / \delta_{1}\right)-m x(t)+D\left(S_{i}-K\right)<0$, 
$\left.(d S / d t)\right|_{D C}>0,\left.(d S / d t)\right|_{A B}=-\left(\mu S_{i} /\left(K_{s}+S_{i}\right) \delta_{1}\right) x(t)-m x(t)<$ 0 . Hence, the system (1) is uniformly bounded. The proof is completed. We denote that the region $\Omega$ is formed by four points $A, B, C$, and $D$.

The equilibrium points of system (1) satisfies the following equation.

$$
\begin{gathered}
-\frac{\mu S}{\left(K_{s}+S\right) \delta_{1}} x-m x+D\left(S_{i}-S\right)=0, \\
\frac{\mu S}{K_{s}+S} x-\left(K_{d}+D\right) x=0 .
\end{gathered}
$$

It can be seen that system (11) has a boundary equilibrium $Q\left(S_{i}, 0\right)$ and a positive equilibrium $H\left(S^{*}, x^{*}\right)$ if $S_{i}-\left(K_{d}+\right.$ $D) K_{s} /\left(\mu-\left(K_{d}+D\right)\right)>0$, where $S^{*}=\left(K_{d}+D\right) K_{s} /(\mu-$ $\left.\left(K_{d}+D\right)\right), x^{*}=D \delta_{1}\left(S_{i}-S^{*}\right) /\left(K_{d}+D+m \delta_{1}\right)$.

In the following, we will analyze the stability of equilibrium $Q\left(S_{i}, 0\right)$ and $H\left(S^{*}, x^{*}\right)$ of system (1). If $S_{i}-\left(K_{d}+\right.$ $D) K_{s} /\left(\mu-\left(K_{d}+D\right)\right)<0$, the positive equilibrium point does not exist, and the boundary equilibrium is stable. That is, we have the following result.

Theorem 8. The equilibrium $Q\left(S_{i}, 0\right)$ is locally asymptotically stable if $R<1$, where $R=S_{i}\left(\mu-\left(K_{d}+D\right)\right) /\left(K_{d}+D\right) K_{s}$. Otherwise, it is unstable.

Proof. The Jacobian matrix $J_{Q}=J\left(S_{i}, 0\right)$ of system (1) at $A$ takes the form of

$$
\left(\begin{array}{cc}
-D & -\frac{\mu S_{i}}{\left(K_{s}+S_{i}\right) \delta_{1}}-m \\
0 & \frac{\mu S_{i}}{K_{s}+S_{i}}-\left(K_{d}+D\right)
\end{array}\right)
$$

It is easy to obtain that the eigenvalues for the matrix $J_{\mathrm{Q}}=$ $J\left(S_{i}, 0\right)$ are $\lambda_{1}=-D<0, \lambda_{2}=\left(\mu S_{i} /\left(K_{s}+S_{i}\right)\right)-\left(K_{d}+D\right)$. For $R<1$, then $\left(\mu S_{i} /\left(K_{s}+S_{i}\right)\right)-\left(K_{d}+D\right)<0$, that is, $\lambda_{2}<0$; hence, $\mathrm{Q}\left(S_{i}, 0\right)$ is locally asymptotically stable. Otherwise, $A\left(S_{i}, 0\right)$ is unstable. This completes the proof.

Theorem 9. The equilibrium point $\mathrm{Q}\left(S_{i}, 0\right)$ is globally asymptotically stable if $R<1$, where $R=S_{i}\left(\mu-\left(K_{d}+D\right)\right) /\left(K_{d}+D\right) K_{s}$.

Proof. Consider the Lyapunov function $V(t)=x(t)$; we can obtain the result immediately.

Theorem 10. If $R>1$, then the positive equilibrium point $H$ is locally asymptotically stable.

Proof. The Jacobian matrix $J_{H}=J\left(S^{*}, x^{*}\right)$ of system (1) at $H$ takes the form of

$$
J_{H}=\left(\begin{array}{cc}
-\frac{\mu K_{s} x^{*}}{\delta_{1}\left(K_{s}+S^{*}\right)^{2}}-D & -\frac{\left(K_{d}+D\right)}{\delta_{1}}-m \\
\frac{\mu K_{s} x^{*}}{\left(K_{s}+S^{*}\right)^{2}} & 0
\end{array}\right) .
$$

The eigenvalue problem for the $J_{H}=J\left(S^{*}, x^{*}\right)$ provides the following characteristic equation:

$$
\lambda^{2}+Q_{1} \lambda+Q_{2}=0
$$

where the coefficients $Q_{1}, Q_{2}$ are

$$
\begin{gathered}
Q_{1}=\frac{\mu K_{s} x^{*}}{\delta_{1}\left(K_{s}+S^{*}\right)^{2}}+D \\
Q_{2}=\frac{\mu K_{s} x^{*}}{\left(K_{s}+S^{*}\right)^{2}}\left(\frac{\left(K_{d}+D\right)}{\delta_{1}}+m\right) .
\end{gathered}
$$

Note that $Q_{1}>0 ; Q_{2}>0$, then, we have that $H$ is locally asymptotically stable.

In the following, we will discuss the global stability of system (1). Firstly, we will give the following lemma.

Lemma 11. Suppose that $\Gamma(T)=(S(t), x(t))$ is a periodic orbit with $T$ of system (1), and $\mathfrak{R}$ is the set which consists of all the points in Phase plane $\Gamma$.

Denote

$$
N=\int_{0}^{T}\left(\frac{\partial f_{1}}{\partial S}(S(t), x(t))+\frac{\partial f_{2}}{\partial x}(S(t), x(t))\right) d t
$$

where $S^{\prime}(t)=f_{1}(S(t), x(t)), x^{\prime}(t)=f_{2}(S(t), x(t))$; then, we can obtain $N<0$.

Proof

$$
\begin{aligned}
N & =\int_{0}^{T}\left[-\frac{\mu K_{s} x}{\delta_{1}\left(K_{s}+S\right)^{2}}-2 D-K_{d}+\frac{\mu S}{K_{s}+S}\right] d t \\
& =\int_{0}^{T}\left[-\frac{\mu K_{s} x}{\delta_{1}\left(K_{s}+S\right)^{2}}-D+\left(\frac{\mu S}{K_{s}+S}-D-K_{d}\right)\right] d t,
\end{aligned}
$$

for $x(t)$ is a period function with $T$, so $\int_{0}^{T}\left(\left(\mu S / K_{s}+S\right)-D-\right.$ $\left.K_{d}\right) d t=\int_{0}^{T} d \ln x(t)=0$; hence,

$$
N=\int_{0}^{T}\left[-\frac{\mu K_{s} x}{\delta_{1}\left(K_{s}+S\right)^{2}}-D\right] d t
$$

It is evident that $N<0$; the proof is completed.

Theorem 12. If $R>1$, the positive equilibrium $H$ of system (1) is globally asymptotically stable.

Proof. From Theorem 10, we know that $H$ is locally stable. According to Lemma 11, we can obtain if there exists periodic solution $(S(t), x(t))$ around $H\left(S^{*}, x^{*}\right)$; then, it is stable for any periodic solution. This is impossible. According to the Poincare-Bendixson theorem, limit set $\omega$ of all orbits must be equilibrium point $H$. This implies that $H\left(S^{*}, x^{*}\right)$ is globally asymptotically stable in $\Omega$. This completes the proof. 


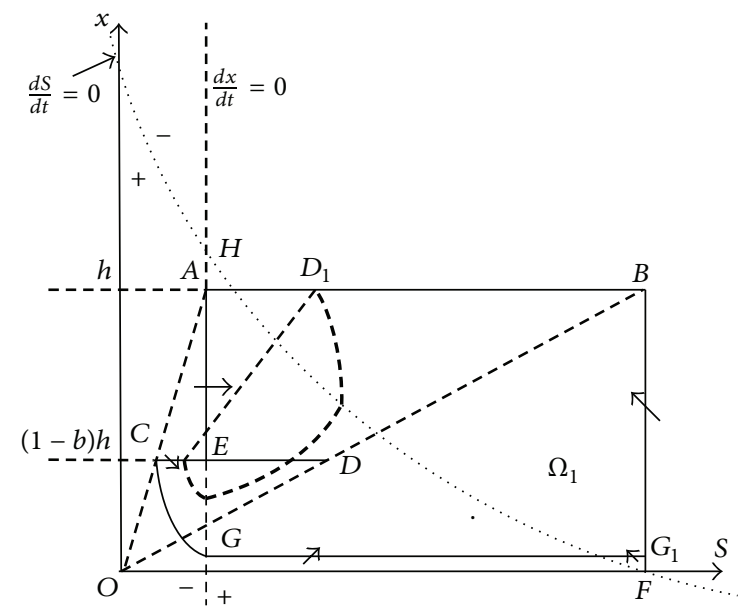

FIgURE 2: Illustration of (2) when $R>1, h<x^{*}, x(0) \leq h$, and $S_{D} \geq S^{*}$.

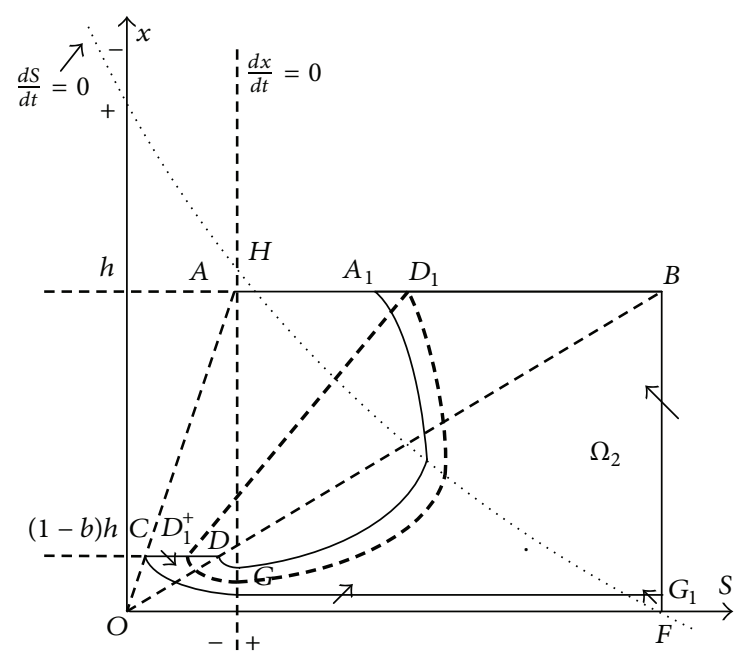

FIGURE 3: Illustration of (2) when $R>1, h<x^{*}, x(0) \leq h$, and $S_{D}<S^{*}$.

\section{Existence and Stability of Periodic Solution of System (2)}

4.1. Existence of Order-1 Periodic Solution. From discussions of the qualitative characteristic of system (2) without the impulsive effects, we can see that if $R>1, H\left(S^{*}, x^{*}\right)$ is globally stable. For the initial points which satisfy $x(0)<x^{*}$ and $\left.(d S / d t)\right|_{\left(S_{0}, x_{0}\right)} \geq 0$, if $h>x^{*}$, then all the solutions of system (2) will tend to the positive equilibrium $\left(S^{*}, x^{*}\right)$ after at most finite times impulsive effects. Therefore, we mainly focus our attention on the cases $h<x^{*}, x(0)<x^{*}$ and $S(0)<S_{i}$.

According to the existence criteria (Theorem 6), our ideas to prove the existence of periodic solution is to construct a closed region such that all the solutions of (2) enter the closed region and retain there. The ideas will be illustrated as follows using Figures 2 and 3.
From Figure 2, we can see that the line $x=h$ interacts with the line $S=S^{*}$ at the point $A\left(S_{A}, h\right)$, where $S_{A}=S^{*}$. The impulsive set $M \subseteq \overline{A B}, \overline{A B}=\left\{(S, x) \mid x=h, S^{*} \leq S \leq S_{i}\right\}$. The impulsive functions $I_{1}$ and $I_{2}$ map the impulsive set $M$ into $N=I(M) \subseteq \overline{C D}, \overline{C D}=\left\{(S, x) \mid x=(1-b) h,(1-b) S^{*} \leq\right.$ $S \leq(1-b) S_{i}$, where $C=\left(S_{C},(1-b) h\right), D=\left(S_{D},(1-b) h\right)$, $S_{C}=(1-b) S^{*}$, and $S_{D}=(1-b) S_{i}$. From the third equation of (2), we know that $S^{*+}=(1-b) S^{*}$ for $x=h$, and furthermore, $S_{C}=(1-b) S_{A}<S_{A}$. Since the straight lines $A C$ and $B D$ satisfy $x=h S / S_{A}$ and $x=h S / S_{i}$, respectively, and both pass through the point $(0,0)$, then it is clear that there are two cases of the point $D$, that is, $\left(K_{d}+D\right) K_{s} /\left(\mu-\left(K_{d}+D\right)\right)=S^{*} \leq S_{D}$ and $S_{D}<S^{*}=\left(K_{d}+D\right) K_{s} /\left(\mu-\left(K_{d}+D\right)\right)$.

Theorem 13. Suppose that $R>1, h<x^{*}, x(0) \leq h$, then system (2) has an order-1 periodic solution; furthermore, this order-1 periodic solution is unique.

Proof. Suppose that $S_{D} \geq S^{*}=\left(K_{d}+D\right) K_{s} /\left(\mu-\left(K_{d}+D\right)\right)$, then the trajectories of (2) starting from the region $\{x(0) \leq h\}$ must interact with the segment $\overline{A B}$. Next, we construct the closed region $\Omega_{1}$. Let $G$ be the intersection point of the line $S=S^{*}$, and the trajectories of (2) starting from the point $C$, let $G_{1}$ be the intersection point of the line $x=x_{G}$, and $S=S_{i}$, let $E$ be the intersection point of the line $S=S^{*}$ and the segment $\overline{C D}$. From the qualitative characteristic of (1), it is easily obtained that $\left.(d S / d t)\right|_{\overline{B G_{1}}}<0,\left.(d x / d t)\right|_{\overline{G G_{1}}}>0$, $\left.(d x / d t)\right|_{\overline{C E}}<0,\left.(d S / d t)\right|_{\overline{A E}}>0$; thus, the closed region $\Omega_{1}$ consists of $\overline{A E}, \overline{E C}, \widehat{C G}, \overline{G G_{1}}, \overline{G_{1} B}$ and $\overline{B A}$ (see Figure 2), where the arc $\widehat{C G}$ is the part of trajectory passing through $C$ and $G$. In addition, there is no singularity in the region $\Omega_{1}$ and all the trajectories satisfying the conditions of the theorem enter the closed region $\Omega_{1}$ and retain there; therefore, from Theorem 6, we know that system (2) has an order-1 periodic solution.

Suppose that $S_{D}<S^{*}=\left(K_{d}+D\right) K_{s} /\left(\mu-\left(K_{d}+D\right)\right)$. Similar to the discussions of the case $S_{D} \geq S^{*}=\left(K_{d}+D\right) K_{s} /\left(\mu-\left(K_{d}+\right.\right.$ $D)$ ), let $A_{1}$ be the intersection point of the line $x=h$ and the trajectories of (2) starting from the point $D$; we can take the $\operatorname{arc} \widehat{D A}_{1}$ as a part of the boundaries of the closed region $\Omega_{2}$. Then, we obtain the closed region $\Omega_{2}$ which consists of $\overline{D C}$, $\widehat{C G}, \overline{G G_{1}}, \overline{G_{1} B}, \overline{B A_{1}}$, and $\widehat{A_{1} D}$ (see Figure 3 ); therefore, from Theorem 6 , we know that system (2) has an order-1 periodic solution. To sum up, the system (2) has an order-1 periodic solution under the condition of Theorem 13. This completes the proof.

Remark 14. If system (2) has an order-1 periodic solution, then the order-1 periodic solution is unique.

We can prove the uniqueness of order-1 periodic solution in system (2) by using the method of successor functions which was introduced in [23], here omitted.

4.2. Stability of Order-1 Periodic Solution. In the following, we will analyze the stability of order-1 periodic solution in system (2). Firstly, we give one lemma to discuss the stability of this periodic solution of system (2). 
Lemma 15. The T-periodic solution $S=\xi(t), x=\eta(t)$ of the following system

$$
\begin{aligned}
& \frac{d S}{d t}=P(S, x), \\
& \frac{d x}{d t}=Q(S, x), \\
& \Delta S=\alpha(S, x), \quad \text { if } \phi(S, x) \neq 0, \\
& \Delta x=\beta(S, x)
\end{aligned}
$$

is orbitally asymptotically stable if the Floquet multiplier $\mu_{2}$ satisfies the condition $\left|\mu_{2}\right|<1$, where

$$
\begin{aligned}
\mu_{2}=\Pi_{k=1}^{q} \Delta_{k} \exp \left[\int _ { 0 } ^ { T } \left(\frac{\partial P}{\partial S}(\xi(t), \eta(t))\right.\right. \\
\left.\left.+\frac{\partial Q}{\partial x}(\xi(t), \eta(t))\right) d t\right],
\end{aligned}
$$

with

$$
\begin{aligned}
\Delta_{k}= & \left(P_{+}\left(\frac{\partial \beta}{\partial x} \frac{\partial \phi}{\partial S}-\frac{\partial \beta}{\partial S} \frac{\partial \phi}{\partial x}+\frac{\partial \phi}{\partial S}\right)\right. \\
& \left.+Q_{+}\left(\frac{\partial \alpha}{\partial S} \frac{\partial \phi}{\partial x}-\frac{\partial \alpha}{\partial x} \frac{\partial \phi}{\partial S}+\frac{\partial \phi}{\partial x}\right)\right) \\
& \times\left(P \frac{\partial \phi}{\partial S}+Q \frac{\partial \phi}{\partial x}\right)^{-1},
\end{aligned}
$$

and $P, Q, \partial \alpha / \partial S, \partial \alpha / \partial x, \partial \beta / \partial S, \partial \beta / \partial x, \partial \phi / \partial S$ and $\partial \phi / \partial x$ are calculated at the point $\left(\xi\left(\tau_{k}\right), \eta\left(\tau_{k}\right)\right), P_{+}=P\left(\xi\left(\tau_{k}^{+}\right), \eta\left(\tau_{k}^{+}\right)\right)$, $Q_{+}=Q\left(\xi\left(\tau_{k}^{+}\right), \eta\left(\tau_{k}^{+}\right)\right) . \phi(S, x)$ is a sufficiently smooth function with $\operatorname{grad} \phi(S, x) \neq 0$, and $\tau_{k}(k \in N)$ is the time of the kth jump. [20].

The proof of this lemma referres to Simeonov and Bainov

In the following, we suppose that this periodic solution of system (2) with period $T$ passes through the points $D_{1}^{+}((1-$ b) $\left.\zeta_{0},(1-b) h\right) \in \overline{C D}$ and $D_{1}\left(\zeta_{0}, h\right) \in \overline{A B}$ (see Figures 2 and 3 ). As the expression and the period of this solution are unknown, we discuss the stability of this periodic solution by Lemma 15. In our case,

$$
\begin{gathered}
P(S, x)=-\frac{\mu S x}{\delta_{1}\left(K_{s}+S\right)}-m x+D\left(S_{i}-S\right), \\
Q(S, x)=\frac{\mu S}{K_{s}+S} x-\left(K_{d}+D\right) x, \\
\alpha(S, x)=-b S, \quad \beta(S, x)=-b x \\
\phi(S, x)=x-h, \\
(\xi(T), \eta(T))=\left(\zeta_{0}, h\right), \\
\left(\xi\left(T^{+}\right), \eta\left(T^{+}\right)\right)=\left((1-b) \zeta_{0},(1-b) h\right) .
\end{gathered}
$$

Then,

$$
\begin{gathered}
\frac{\partial P}{\partial S}=-\frac{\mu K_{s} x}{\delta_{1}\left(K_{s}+S\right)^{2}}-D, \quad \frac{\partial Q}{\partial x}=\frac{\mu S}{K_{s}+S}-\left(K_{d}+D\right), \\
\frac{\partial \alpha}{\partial S}=-b, \quad \frac{\partial \alpha}{\partial x}=0, \quad \frac{\partial \beta}{\partial S}=0, \\
\frac{\partial \beta}{\partial x}=-b, \quad \frac{\partial \phi}{\partial S}=0, \quad \frac{\partial \phi}{\partial x}=1, \\
\Delta_{k}=\left(P_{+}\left(\frac{\partial \beta}{\partial x} \frac{\partial \phi}{\partial S}-\frac{\partial \beta}{\partial S} \frac{\partial \phi}{\partial x}+\frac{\partial \phi}{\partial S}\right)\right. \\
\left.+Q\left(\frac{\partial \alpha}{\partial S} \frac{\partial \phi}{\partial x}-\frac{\partial \alpha}{\partial x} \frac{\partial \phi}{\partial S}+\frac{\partial \phi}{\partial x}\right)\right) \\
\times\left(P \frac{\partial \phi}{\partial S}+Q \frac{\partial \phi}{\partial x}\right)^{-1} \\
=\frac{Q_{+}\left(\xi\left(T^{+}\right), \eta\left(T^{+}\right)\right)(1-b)}{Q(\xi(T), \eta(T))} \\
\times\left[\frac{\mu \zeta_{0}}{K_{s}+\zeta_{0}}-\left(K_{d}+D\right)\right]^{-1} \cdot \\
=(1-b)^{2}\left[\frac{\mu(1-b) \zeta_{0}}{K_{s}+(1-b) \zeta_{0}}-\left(K_{d}+D\right)\right]
\end{gathered}
$$

Set $N(t)=(\partial P / \partial S)(\xi(t), \eta(t))+(\partial Q / \partial x)(\xi(t), \eta(t))$; then;

$$
\begin{aligned}
& \mu_{2}= \Delta_{1} \exp \left[\int_{0}^{T}\left(\frac{\partial P}{\partial S}(\xi(t), \eta(t))+\frac{\partial Q}{\partial x}(\xi(t), \eta(t))\right) d t\right] \\
&=\left((1-b)^{2}\left[\frac{\mu(1-b) \zeta_{0}}{K_{s}+(1-b) \zeta_{0}}-\left(K_{d}+D\right)\right]\right. \\
&\left.\times\left[\frac{\mu \zeta_{0}}{K_{s}+\zeta_{0}}-\left(K_{d}+D\right)\right]^{-1}\right) \exp \left[\int_{0}^{T} N(t) d t\right] .
\end{aligned}
$$

Because $(S(t), x(t))$ is a periodic solution of system (2), we know that $\int_{0}^{T} N(t) d t<0$, by the proof of Lemma 11, that is, $\exp \left[\int_{0}^{T} N(t) d t\right]<1$. Obviously, $\left|\mu_{2}\right|<1$ if $\mid(1-b)^{2}[(\mu(1-$ b) $\left.\left.\zeta_{0} /\left(K_{s}+(1-b) \zeta_{0}\right)\right)-\left(K_{d}+D\right)\right] /\left[\left(\mu \zeta_{0} /\left(K_{s}+\zeta_{0}\right)\right)-\left(K_{d}+D\right)\right] \mid<$ 1 . Therefore, we have the following theorem.

Theorem 16. If $R>1, h<x^{*}, x(0) \leq h$ and $\mid(1-b)^{2}[(\mu(1-$ b) $\left.\left.\zeta_{0} /\left(K_{s}+(1-b) \zeta_{0}\right)\right)-\left(K_{d}+D\right)\right] /\left[\left(\mu \zeta_{0} /\left(K_{s}+\zeta_{0}\right)\right)-\left(K_{d}+D\right)\right] \mid<$ 1 , then the order-1 periodic solution of system (2) is orbitally asymptotically stable.

4.3. Order-2 Periodic Solution. From Theorem 13, we know that system (2) has an order-1 periodic solution. In this subsection, we will discuss the existence of order- 2 periodic solution.

Suppose that $(\bar{S}, \bar{x})$ is a periodic solution of system (2), then $\left(\overline{S_{0}},(1-b) h\right) \in N \subseteq \overline{C D}$ and $\left(\overline{S_{1}}, h\right) \in M \subseteq \overline{A B}$. It is 


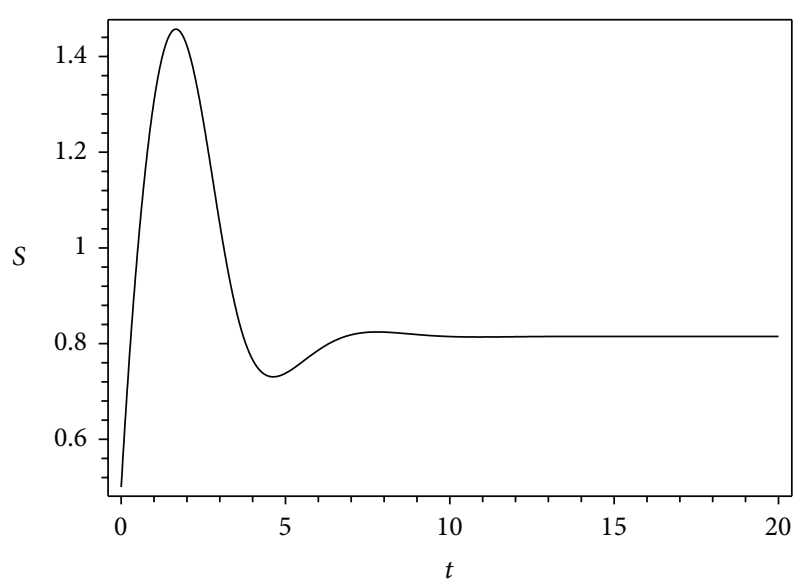

(a)

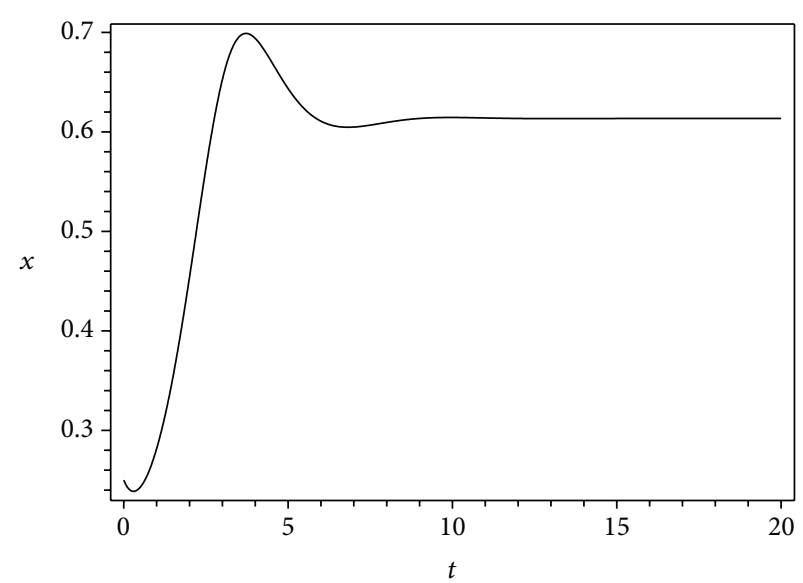

(b)

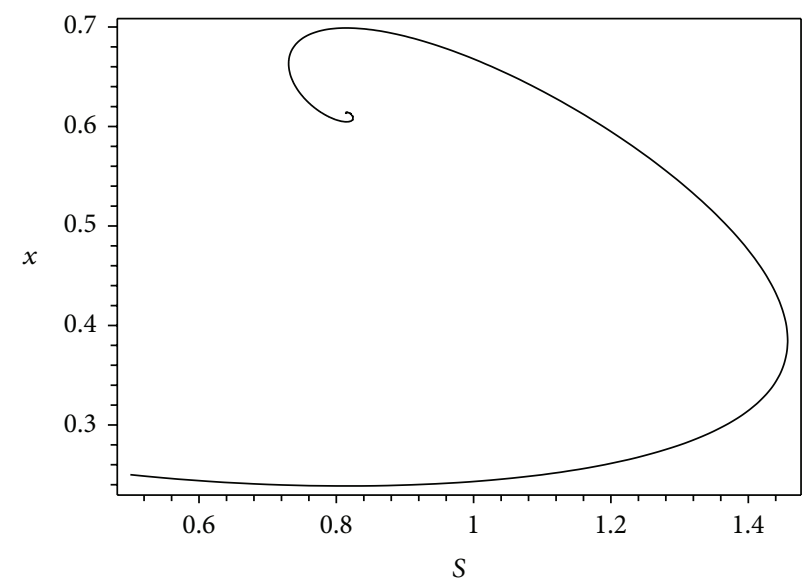

(c)

Figure 4: Time series and phase portrait of system (2) with $\delta_{1}=0.6, K_{s}=2, S_{i}=4.7, D=0.4, \mu=3.8, K_{d}=0.7, b=0.8, S_{0}=0.5, x_{0}=0.25$, and $h=0.78>S^{*}$.

easy to obtain that $\overline{S_{0}}<\overline{S_{1}}$ for $S^{+}=(1-b) S$ by the third equation of system (2). Let $(S, x)$ be the arbitrary solution of system (2) starting from the point $\left(S_{0}^{+},(1-b) h\right)$, and let the first interaction point of trajectory and the set $M(x=h)$ be $\left(S_{1}, h\right)$ and let the corresponding interaction points of every impulse be $\left(S_{2}, h\right),\left(S_{3}, h\right), \ldots$, respectively. Therefore, under the effect of impulsive function $I$, the corresponding points after impulse are $\left(S_{1}^{+},(1-b) h\right),\left(S_{2}^{+},(1-b) h\right), \ldots$, respectively.

By the qualitative analysis of system (2), if there exists the $i$ th impulse effect such that $S_{j}^{+} \geq S^{*}$ for all $j>i \geq 1$, then the sequence $S_{j}^{+}$will be monotone, that is, $S_{j}^{+} \geq S_{j+1}^{+} \geq S_{j+2}^{+} \geq$ $\cdots \geq S^{*}$ or $S^{*} \leq S_{j}^{+} \leq S_{j+1}^{+} \leq S_{j+2}^{+} \leq \cdots$. In this case, system (2) has no order $k(k \geq 2)$ periodic solution. Therefore, if system (2) has order $k(k \geq 2)$ periodic solution, there must exist a $S_{j}^{+}$such that $S_{j}^{+}<S^{*}$. Besides, when $b>1-\left(S^{*} / S_{i}\right)$, we can know that $S_{j}^{+}<S^{*}$ for $j=1,2, \ldots$. So, in the following, we mainly discuss the existence of order-2 periodic solution for $b>1-\left(S^{*} / S_{i}\right)$.
If $S_{0}^{+}=S_{1}^{+}$, then system (2) has an order-1 periodic solution.

If $S_{0}^{+} \neq S_{1}^{+}$, then $S_{0}^{+}<S_{1}^{+}$or $S_{0}^{+}>S_{1}^{+}$. Suppose that $S_{0}^{+}<S_{1}^{+}$, for $b>1-\left(S^{*} / S_{i}\right)$ and the qualitative properties of system (2), we know that $S_{2}^{+}<S_{1}^{+}$and $S_{2}^{+} \leq S_{0}^{+}<S_{1}^{+}$or $S_{0}^{+} \leq S_{2}^{+}<S_{1}^{+}$. Therefore, there exists an order-2 periodic solution if $S_{2}^{+}=S_{0}^{+}$ holds.

If $S_{2}^{+}<S_{0}^{+}<S_{1}^{+}$holds, we can obtain

(i) $(1-b) S^{*}<\cdots<S_{2 k}^{+}<\cdots<S_{4}^{+}<S_{2}^{+}<S_{0}^{+}<S_{1}^{+}<$ $S_{3}^{+}<S_{5}^{+}<\cdots<S_{2 k+1}^{+}<\cdots<S^{*}$.

While if $S_{0}^{+}<S_{2}^{+}<S_{1}^{+}$holds, we have

(ii) $(1-b) S^{*} \leq S_{0}^{+}<S_{2}^{+}<S_{4}^{+}<\cdots<S_{2 k}^{+}<\cdots<S_{2 k+1}^{+}<$ $\cdots<S_{5}^{+}<S_{3}^{+}<S_{1}^{+}<S^{*}$.

The same discussion for the case $S_{0}^{+}>S_{1}^{+}$, that is, we have $S_{1}^{+}<S_{2}^{+}<S_{0}^{+}$or $S_{1}^{+}<S_{0}^{+}<S_{2}^{+}$.

If $S_{1}^{+}<S_{0}^{+}<S_{2}^{+}$holds, we have 


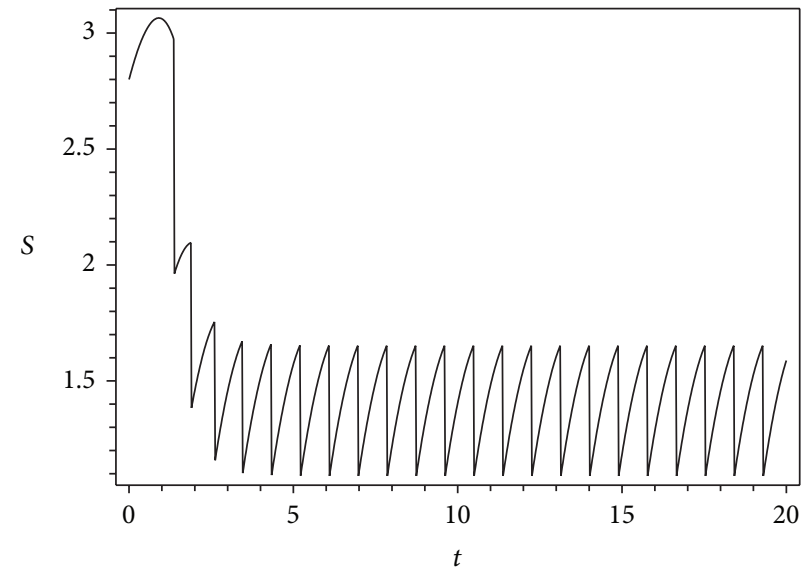

(a)

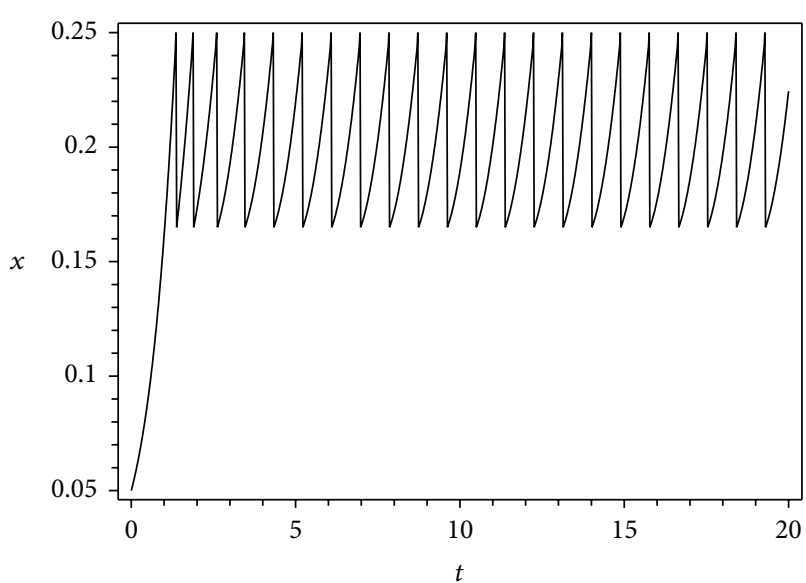

(b)

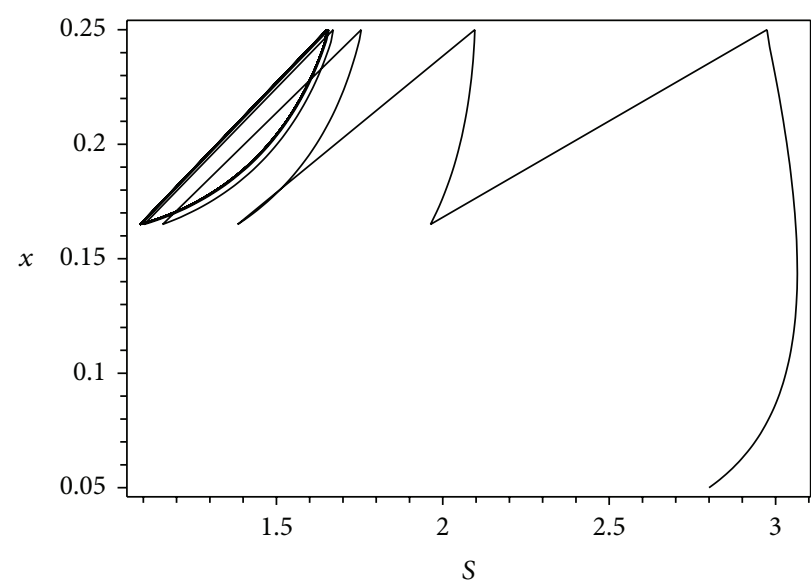

(c)

Figure 5: Time series and phase portrait of system (2) with $\delta_{1}=0.6, K_{s}=2, S_{i}=4.7, D=0.4, \mu=3.8, K_{d}=0.7, b=0.34, S_{0}=2.8, x_{0}=0.05$, $h=0.25<S^{*}$.

(iii) $(1-b) S^{*}<\cdots<S_{2 k+1}^{+}<\cdots<S_{5}^{+}<S_{3}^{+}<S_{1}^{+}<S_{0}^{+}<$ $S_{2}^{+}<S_{4}^{+}<\cdots<S_{2 k}^{+}<\cdots<S^{*}$.

While if $S_{1}^{+}<S_{2}^{+}<S_{0}^{+}$holds, we have

(iv) $(1-b) S^{*} \leq S_{1}^{+}<S_{3}^{+}<S_{5}^{+}<\cdots<S_{2 k+1}^{+}<\cdots<S_{2 k}^{+}<$ $\cdots<S_{4}^{+}<S_{2}^{+}<S_{0}^{+}<S^{*}$.

If the case of (ii) and (iv) holds, system (2) has an order-1 periodic solution. It follows from (i) (or (iii)) that $\lim _{k \rightarrow \infty} S_{2 k}^{+}=S_{0}^{+*}, \lim _{k \rightarrow \infty} S_{2 k+1}^{+}=S_{1}^{+*}$, which implies that system (2) exists with an order-2 periodic solution. Similar to the previous discussion and the proof of Proposition 3.2 in [24], we can obtain that there is no order $k(k \geq 3)$ periodic solution in system (2) for $b>1-\left(S^{*} / S_{i}\right)$.

Remark 17. Since the exact expression of the solution to system (2) is unknown, it is difficult to give the sufficient conditions to guarantee that the system (2) has an order-2 periodic solution or not.

\section{Numerical Analysis and Discussion}

In this paper, a mathematical model of cheese whey fermentation for single-cell protein production with impulsive state feedback control is proposed. We investigated the qualitative characteristic of the system without impulsive effect and show that the system is globally asymptotical stability. It is shown that the system with impulsive state feedback control has an order-1 periodic solution, and sufficient conditions for existence and stability of order-1 periodic solution are also obtained. The case, in which it is possible that there is an order- 2 periodic solution, is also observed; furthermore, it is also pointed out that the system has no order $k(k \geq 3)$ periodic solution for $b>1-\left(S^{*} / S_{i}\right)$. These results show that the cheese whey fermentation system for single-cell protein production with impulsive state feedback control either tends to a stable state or has a periodic solution.

To verify the theoretical results obtained in this paper, we give the numerical simulations of system (2). Let $\delta_{1}=0.6$, $K_{s}=2, S_{i}=4.7, D=0.4, \mu=3.8$ and $K_{d}=0.7$; then, we have $S^{*}=0.8148, x^{*}=0.6135$. If $b=0.8, h=0.78>S^{*}, S_{0}=0.5$, and $x_{0}=0.25$, then the system (2) tends to the positive equilibrium point, which can be seen in Figure 4 . Figure 4 shows that no impulse occurs when $h>S^{*}$. Figure 5 gives the time series and phase portrait when $b=0.34, h=0.25<S^{*}$, $S_{0}=2.8$, and $x_{0}=0.05$ and show the trajectory tends to be 


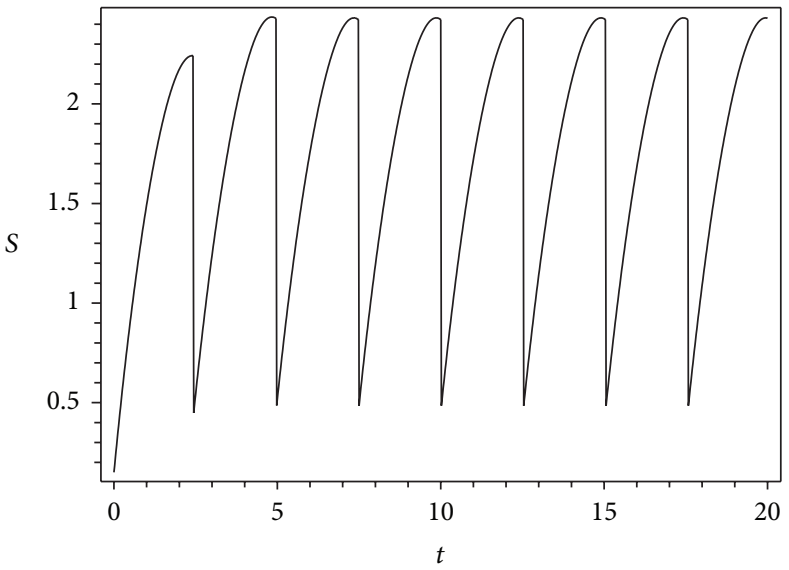

(a)

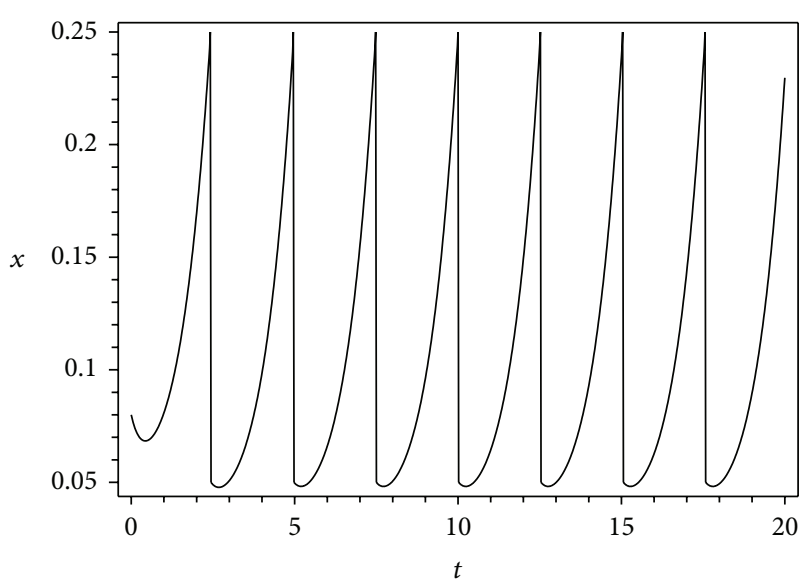

(b)

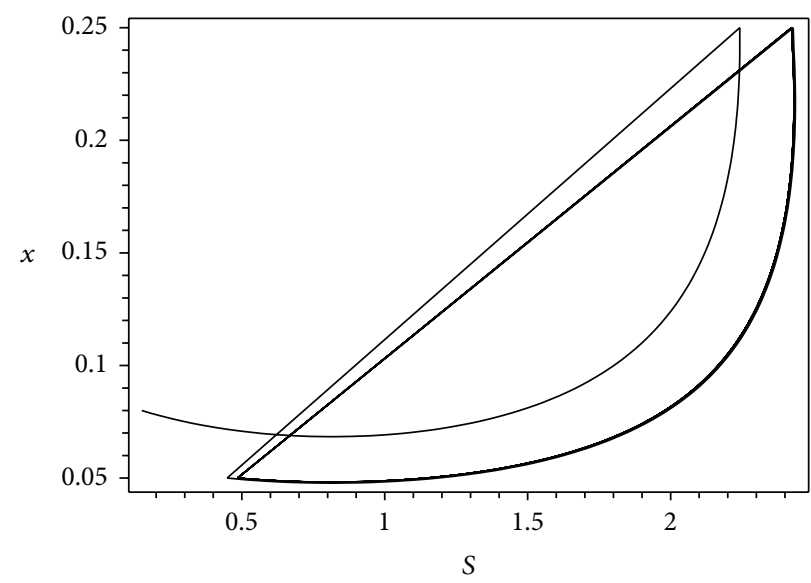

(c)

FiguRE 6: Time series and phase portrait of system (2) with $\delta_{1}=0.6, K_{s}=2, S_{i}=4.7, D=0.4, \mu=3.8, K_{d}=0.7, b=0.8, S_{0}=0.15, x_{0}=0.08$, $h=0.25<S^{*}$.

periodic. Figure 6 is the time series and phase portrait when $b=0.8, h=0.25<S^{*}, S_{0}=0.15$, and $x_{0}=0.08$. Figures 5 and 6 show different positions of the periodic solution under different parameter value $b$ and different initial values. Furthermore, the phase portrait of Figure 5 indicates that the microorganism concentration always keeps increasing, but Figure 6 indicates that the microorganism concentration firstly decreases and then begins to increase. Therefore, a different control parameter $b$ can result in different bioprocesses and different efficiencies of microorganism culture. Which will give a conclusion theoretically to the researchers in the field of single-cell protein production. Researchers should give suitable control parameter $b$ and appropriate initial concentration in order to obtain a steady and optimal production.

In conclusion, we give a mathematical conclusion to the researchers in the field as follows: researchers of cheese whey fermentation for single-cell protein production should give suitable feedback state (the value of $h$ ), control parameters (b), and appropriate initial concentration of microorganism and substrate in order to obtain a steady and optimal production.

\section{Acknowledgments}

This paper supported by National Natural Science Foundation of China (11171284, 31272653), Fujian Provincial Natural Science Foundation of China (2012J01012), the Fujian Provincial Education Foundation (JA12198), and the Scientific Research Foundation of Jimei University of China (ZC2011003).

\section{References}

[1] G. Emtiazi, Z. Etemadifar, and M. Tavassoli, "A novel nitrogenfixing cellulytic bacterium associated with root of corn is a candidate for production of single cell protein," Biomass and Bioenergy, vol. 25, no. 4, pp. 423-426, 2003.

[2] R. B. Vasey and K. A. Powell, "Single-cell protein," Biotechnology and Genetic Engineering Reviews, vol. 2, pp. 285-311, 1984.

[3] R. D. MacElroy and D. Wang, "Waste recycling issues in bioregenerative life support," Advances in Space Research, vol. 9, no. 8, pp. 75-84, 1989.

[4] J. R. Mielenz, "Ethanol production from biomass: technology and commercialization status," Current Opinion in Microbiology, vol. 4, no. 3, pp. 324-329, 2001. 
[5] M. I. Rajoka, S. H. Khan, M. A. Jabbar, M. S. Awan, and A. S. Hashmi, "Kinetics of batch single cell protein production from rice polishings with Candida utilis in continuously aerated tank reactors," Bioresource Technology, vol. 97, no. 15, pp. 1934-1941, 2006.

[6] E. B. Kurbanoglu and O. F. Algur, "Single-cell protein production from ram horn hydrolysate by bacteria," Bioresource Technology, vol. 85, no. 2, pp. 125-129, 2002.

[7] R. El-Saadany, H. Khalaf, H. El-Manawaty, and F. Salom, “The production of single cell protein from agricultural wastes by fungi," Acta Aliment Acad Sci Hung, vol. 17, no. 4, pp. 376-377, 1988.

[8] A. E. Ghaly, M. Kamal, and L. R. Correia, "Kinetic modelling of continuous submerged fermentation of cheese whey for single cell protein production," Bioresource Technology, vol. 96, no. 10, pp. 1143-1152, 2005.

[9] A. E. Ghaly and A. A. El-Taweel, "Kinetic modelling of continuous production of ethanol from cheese whey," Biomass and Bioenergy, vol. 12, no. 6, pp. 461-472, 1997.

[10] K. Schügerl and K. H. Bellgardt, Eds., Bioreaction Engineering: Modeling and Control, Springer, Berlin, Germany, 2000.

[11] S. Sun and L. Chen, "Dynamic behaviors of Monod type chemostat model with impulsive perturbation on the nutrient concentration," Journal of Mathematical Chemistry, vol. 42, no. 4, pp. 837-847, 2007.

[12] P. de Leenheer and H. Smith, "Feedback control for chemostat models," Journal of Mathematical Biology, vol. 46, no. 1, pp. 4870, 2003.

[13] G. Fu, W. Ma, and S. Ruan, "Qualitative analysis of a chemostat model with inhibitory exponential substrate uptake," Chaos, Solitons \& Fractals, vol. 23, no. 3, pp. 873-886, 2005.

[14] K. Sun, A. Kasperski, Y. Tian, and L. Chen, "Modelling and optimization of a continuous stirred tank reactor with feedback control and pulse feeding," Chemical Engineering and Processing, vol. 50, no. 7, pp. 675-686, 2011.

[15] H. Guo and L. Chen, "Periodic solution of a chemostat model with Monod growth rate and impulsive state feedback control," Journal of Theoretical Biology, vol. 260, no. 4, pp. 502-509, 2009.

[16] Z. Zhao, L. Yang, and L. Chen, "Impulsive state feedback control of the microorganism culture in a turbidostat," Journal of Mathematical Chemistry, vol. 47, no. 4, pp. 1224-1239, 2010.

[17] Y. Tian, A. Kasperski, K. Sun, and L. Chen, "Theoretical approach to modelling and analysis of the bioprocess with product inhibition and impulse effect," BioSystems, vol. 104, no. 2-3, pp. 77-86, 2011.

[18] Z. Li, L. Chen, and Z. Liu, "Periodic solution of a chemostat model with variable yield and impulsive state feedback control," Applied Mathematical Modelling, vol. 36, no. 3, pp. 1255-1266, 2012.

[19] G. Zeng, L. Chen, and L. Sun, "Existence of periodic solution of order one of planar impulsive autonomous system," Journal of Computational and Applied Mathematics, vol. 186, no. 2, pp. 466-481, 2006.

[20] P. E. Simeonov and D. D. Bainov, "Orbital stability of periodic solutions autonomous systems with impulse effect," International Journal of Systems Science, vol. 19, no. 12, pp. 2562-2585, 1988.

[21] V. Laksmikantham, D. D. Bainov, and P. S. Simeonov, Theory of Impulsive Differential Equations, World Scientific, Singapore, 1989.
[22] D. H. Griffel, Applied Functional Analysis, Ellis Horwood, Chichester, UK, 1981.

[23] B. Liu, Y. Tian, and B. Kang, "Dynamics on a Holling II predator-prey model with state-dependent impulsive control," International Journal of Biomathematics, vol. 5, no. 3, article 1260006, 18 pages, 2012.

[24] G. Jiang, Q. Lu, and L. Qian, "Complex dynamics of a Holling type II prey-predator system with state feedback control," Chaos, Solitons \& Fractals, vol. 31, no. 2, pp. 448-461, 2007. 


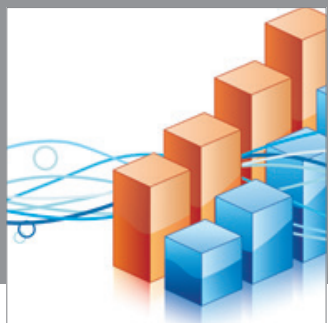

Advances in

Operations Research

mansans

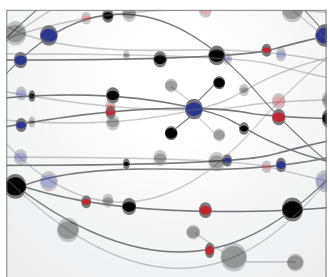

The Scientific World Journal
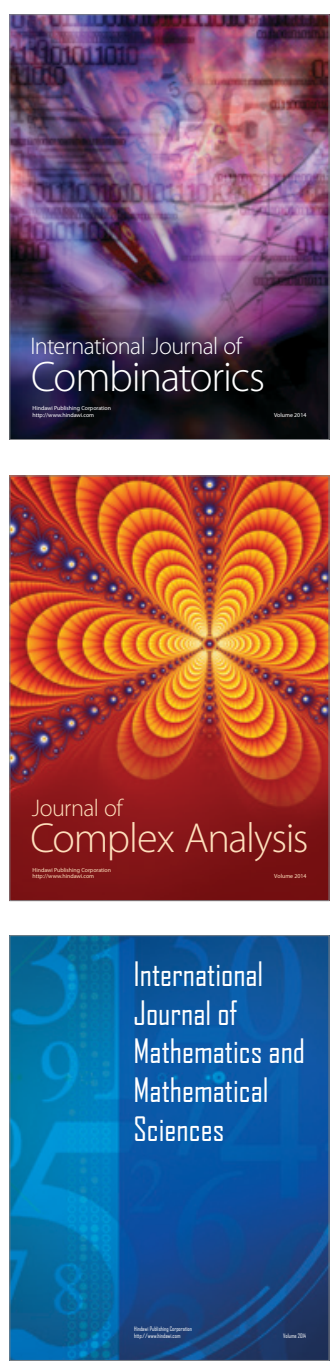
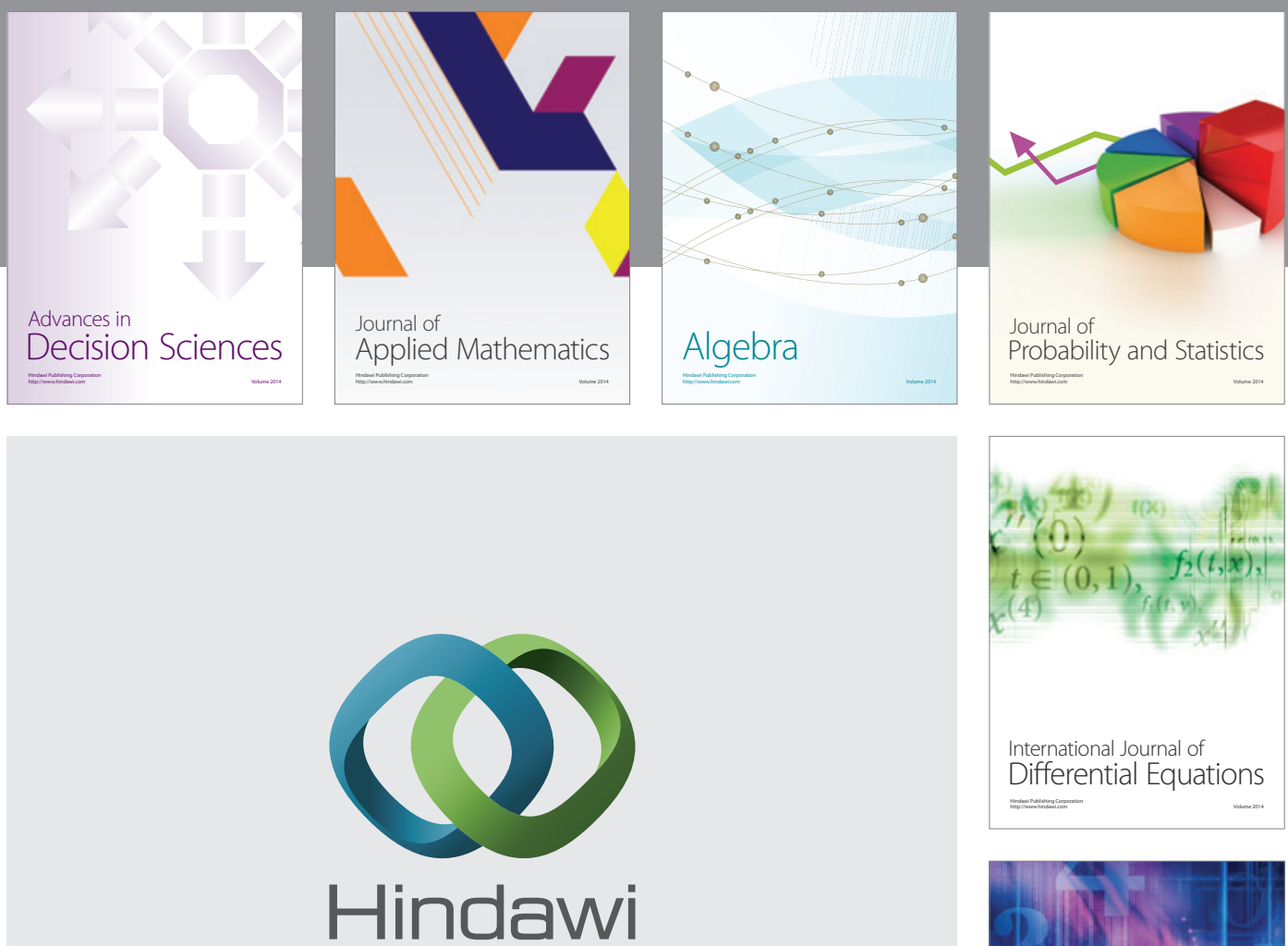

Submit your manuscripts at http://www.hindawi.com
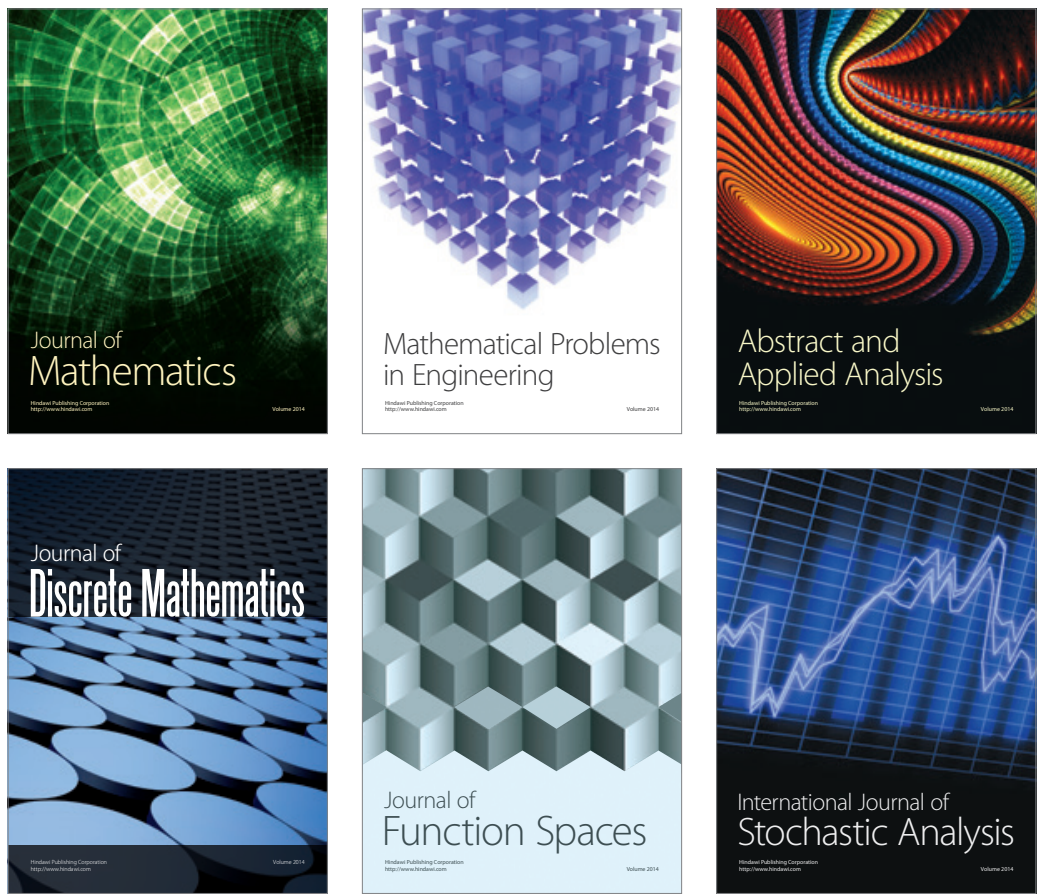

Journal of

Function Spaces

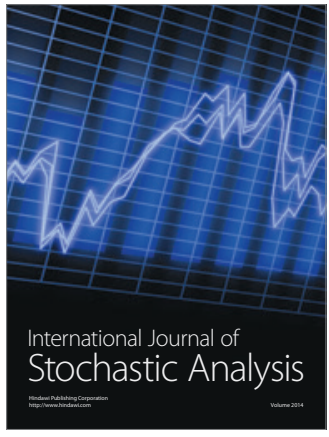

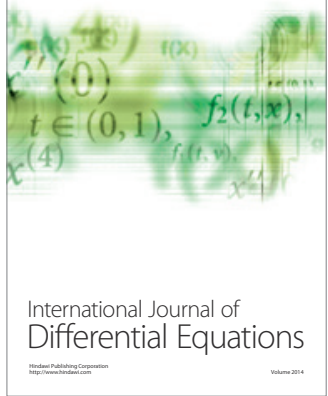
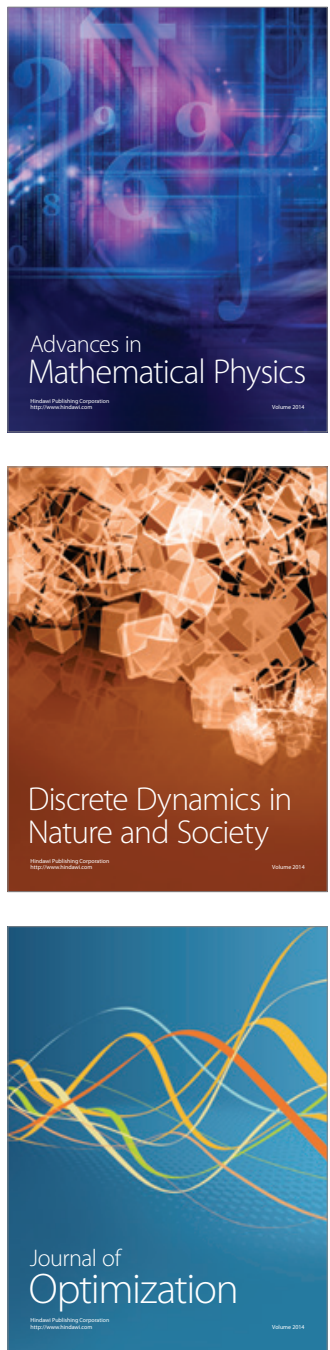